www.jmscr.igmpublication.org

Impact Factor 5.84

Index Copernicus Value: 71.58

ISSN (e)-2347-176x ISSN (p) 2455-0450

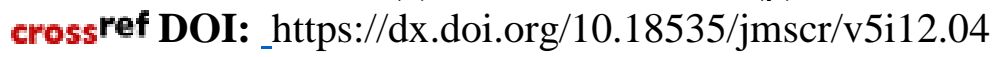

Journal Of Medical Science And Clinical Research

\title{
A Profile of Spontaneous Bacterial Peritonitis and Its Variants in Cases of Liver Cirrhosis
}

\author{
Authors \\ Dr Rohan Yewale ${ }^{1}$, Dr Jeyhan Dhabhar ${ }^{2}$, Dr Jaishree Ghanekar ${ }^{3}$ \\ ${ }^{1}$ Senior Resident, ${ }^{2}$ Resident, ${ }^{3}$ Prof. \& Head of Dept. Internal Medicine \\ MGM Medical College \& Hospital, Navi Mumbai
}

\begin{abstract}
Background: Spontaneous bacterial peritonitis is a complication of decompensated liver cirrhosis ${ }^{1}$ notorious for its high recurrence rates and is frequently associated with short term as well as long term mortality in the affected patients.

Objective: To analyse the incidence, clinical and bacteriological profile of patients with spontaneous bacterial peritonitis or its variants such as culture negative neutrocytic ascites and bacterascites in cases of liver cirrhosis. Patients with SBP or its variants were compared to patients with Non SBP Ascites in order to assess the predictive factors for the development of SBP or its variants and thereby relate the in hospital mortality in these patients with various possible parameters.

Methods: The present study was a prospective, cross sectional, comparative study conducted among 60 patients with ascites due to liver cirrhosis fulfilling the proposed inclusion and exclusion criteria, admitted at MGM Medical College and Hospital, Navi Mumbai, between November 2014 and November 2016. Ascitic fluid routine and culture analysis with antibiotic sensitivity testing along with other routine blood and urine investigations were performed at an NABL (National Accreditation Board for Testing and Calibration Laboratories) certified laboratory at MGM Medical College and Hospital, Navi Mumbai.

Results: Our study demonstrated an in hospital mortality of $21.67 \%$ amongst all patients and $20 \%$ amongst patients with SBP or its variants similar to the study by Nakul Kadam et al (17\%). Our study also revealed that variables such as Child Pugh Grade C, MELD Na score > 30, anemia, leukocytosis, coagulopathy or deranged PT INR, hyperbilirubinemia, hyponatremia, AKI, hepatic encephalopathy, refractory Ascites, hepatorenal Syndrome and absence of albumin transfusion during hospitalization were significantly associated with mortality.

Conclusion: Spontaneous bacterial peritonitis is a frequent and unprecedentedly mortal complication that is often undiagnosed in patients of liver cirrhosis with ascites unless there is a high degree of suspicion. Our study corroborates previous postulates on this subject with regard to predictive factors for development of SBP \& associated mortality. However, being a small sample study, the results cannot be subjected to a large scale population.

Keywords: Ascites, Hepatic encephalopathy, Hepatorenal syndrome, Liver cirrhosis, Spontaneous bacterial peritonitis.
\end{abstract}

\section{Introduction}

Cirrhosis $^{2}$ is a diffuse process, characterized by fibrosis and conversion of normal liver architecture into structurally abnormal nodules and in most cases, the process follows or is accompanied by hepatocellular necrosis. This in 
turn leads to development of intrahepatic, sinusoidal portal hypertension and end stage liver disease $^{3}$. Ascites is a known complication of cirrhosis and portal hypertension. Usually it is sterile, but when it gets infected by bacteria then the condition is termed as Spontaneous Bacterial peritonitis. (SBP) $)^{4,5}$. It is defined as a primary or spontaneous infection of ascitic fluid in the absence of any contiguous source of infection (e.g. intestinal perforation, intra-abdominal abscess) or an identifiable intra-abdominal inflammatory focus (e.g. acute pancreatitis, cholecystitis) ${ }^{6,7,8,9,10}$. SBP can be diagnosed by a simple bedside procedure such as abdominal paracentesis revealing an elevated ascitic fluid neutrophil count $\left(\geq 250\right.$ cells $\left./ \mathrm{mm}^{3}\right)$ and/or a positive ascitic fluid culture ${ }^{11}$. There has been substantial research on this lethal complication of decompensated liver cirrhosis in the past. A number of cross sectional as well as prospective studies have been completed in the western countries over the past few decades focussing on the clinical, diagnostic as well as therapeutic profile of spontaneous bacterial peritonitis. However, population based data and research on this clinical entity in the Indian subcontinent has been surprisingly limited. Cirrhotic patients with SBP are always in a decompensated status and thus may also present with refractory ascites or other complications such as hyponatremia, hepatic encephalopathy, and coagulopathy or hepatorenal syndrome. Due to this overlap it is difficult to know whether the mortality in these instances is due primarily to the infection itself or to the decompensated status of liver. A number of prognostic scoring systems such as the Child Pugh and the MELD $\mathrm{Na}$ score which incorporate important biochemical parameters such as serum creatinine, serum bilirubin, prothrombin time, serum albumin and relevant clinical parameters such as the degree of ascites and stage of encephalopathy, have been established in the past. However, there has been limited research on the prognostic significance of these individual parameters and their association with mortality in patients with decompensated liver cirrhosis. Thus, the real impact of the first SBP event on mortality for cirrhotic patients with ascites needs to be thoroughly evaluated.

\section{Materials and Methods}

The present study was a prospective, cross sectional, comparative study conducted among 60 patients with ascites due to liver cirrhosis fulfilling the proposed inclusion and exclusion criteria, admitted at MGM Medical College and Hospital, Navi Mumbai. The study was conducted over a period of two years between November 2014 and November 2016.

Inclusion criteria:1) Both male and female 2) Age group 18 to 70 years. 3) Ascites only due to liver cirrhosis of any etiology (eg. Alcoholic Liver Disease, Post-Necrotic Liver Cirrhosis, Autoimmune hepatitis, Wilson's Disease, Haemochromatosis, Alpha1anti-trypsin deficiency, Glycogen storage diseases, cryptogenic cirrhosis etc.)

Exclusion criteria:1) Age above 70 years and below 25 years. 2) Discharge / Death after $1^{\text {st }}$ diagnostic tap or before starting antibiotic therapy or discharge within 7 days of hospitalization. 3) Non-cirrhotic ascites. 4) Secondary bacterial peritonitis: Ascites secondary to an intraabdominal pathology such as ovarian cyst, pancreatitis, ruptured intra-abdominal abscess, intestinal perforation etc.

\section{Methodology}

Patient's general information was noted. A detailed history was taken. A detailed general physical examination along with signs of hepatocellular failure were sought and noted. Cirrhosis and ascites was clinically diagnosed and confirmed by ultrasound of abdomen. Routine investigations like CBC, LFT, RFT, serum electrolytes, urine routine, microscopy and PT INR were estimated in all cases. In all suspected patients of spontaneous bacterial peritonitis or its variants, a diagnostic abdominal paracentesis was performed on admission before starting antibiotic medication either for prophylactic or therapeutic purpose. Ascitic fluid was subsequently sent for 
routine, microscopic and culture examination. Patients were also evaluated at the time of paracentesis to determine the Child Pugh score for estimating the severity of liver disease. In addition to the above grading, every patient's MELD Na (Model for End Stage Liver Disease - Sodium) score was also calculated using the MELD Na calculator application available on internet.

\section{Results}

Mean age of patients in the study was $48.62 \pm 11.36$ years. Majority of the patients were in the 40-49 years age group. Alcohol was the predominant etiology of liver cirrhosis in 41/60 (68.33\%) of all patients and in 20/33 (60.60\%) patients with SBP or its variants, followed by Hepatitis B in 10/60 (16.67\%) and so on as elaborated in Graph 1. Abdominal distension was the presenting complaint of all patients followed by abdominal pain in 32/60(53\%), swelling in b/l feet in $32 / 60(53 \%)$, fever in $27 / 60(45 \%)$, jaundice in $18 / 60(30 \%)$, other non-specific complaints in $13 / 60(21 \%)$, GI bleeding manifestations in the form of hematemesis and malena in 12/60(20\%), altered sensorium in $9 / 60(15 \%)$ and oliguria in $4 / 60(6 \%)$ of all patients. When patients of SBP or its variants were compared with patients with Non SBP ascites, the presenting complaints of fever ( $p$ value $=0.006)$ and altered sensorium $(\mathrm{p}$ value $=$ 0.027 ) were found to be statistically significant in patients of SBP or its variants (graph 2). In hospital mortality amongst all patients was 14/60 (21.67\%) out of which 12/60 (20\%) of all patients had SBP or its variants. In $8 / 14$ of these patients $(61.54 \%)$ mortality was due to Culture positive spontaneous bacterial peritonitis; mortality amongst all patients with culture positive spontaneous bacterial peritonitis was $8 / 10(80 \%)$. 4/14 (30.76\%) mortality was due to Culture negative neutrocytic ascites; mortality amongst all patients with CNNA was 4/22 (18.18\%). 2/14 (7.69\%) mortality was due to non SBP ascites; mortality amongst non SBP ascites was 2/27 (3.7\%). Refractory ascites was present in 19/60 (31.67\%) patients and 15/19 (78.94\%) patients with refractory ascites had SBP or its variants. Hepatic encephalopathy was present in $8 / 60$ $(24.24 \%)$ patients and $7 / 8(87.5 \%)$ patients with hepatic encephalopathy also had SBP or its variants. Hepatorenal syndrome was present in $7 / 60(21.21 \%)$ patients and 4/7 (57.14\%) patients had SBP or its variants. When patients with SBP or its variants were compared to Non SBP ascites, Hepatic encephalopathy ( $\mathrm{p}$ value $=0.047$ ), Refractory ascites ( $\mathrm{p}$ value $=0.011$ ) and in hospital mortality ( $p$ value $=0.008$ ) were found to be statistically significant complications associated with SBP or its variants (graph 3). E.coli was the predominant organism found on culture in 5/11 (45.45\%) patients (10 culture positive SBP and 1 Bacterascites), followed by Enterococcus species found in 2/11 (18.18\%) patients followed by Klebsiella, Pseudomonas, Coagulase negative staphylococcus aureus found in $1 / 11$ (9.09\%) each. 23/60 (38.33\%) patients received Ceftriaxone as the initial antibiotic, followed by Cefotaxime in 17/60 patients (28.33\%), Norfloxacin in 15/60 (25\%) patients. Depending upon culture report and patient progress, other antibiotics used were piperacillin + tazobactam, cefaperazone + sulbactam in $2 / 60$ (3.33\%) patients each and ticarcillin + clavulanate in $1 / 60(1.67 \%)$ patient. Mortality was significantly lower in patients with MELD Na score $<30$ as compared to patients with score $>30$ (p value < 0.001) (graph 4). In Hospital Mortality was statistically more significant in the group of patients who had subsequently developed these complications such as hepatic encephalopathy ( $p$ value $<0.001$ ), hepatorenal syndrome ( $\mathrm{p}$ value $<$ $0.001)$ and refractory ascites $(\mathrm{p}$ value $=0.024)$ (graph 5). 
Graph 1:

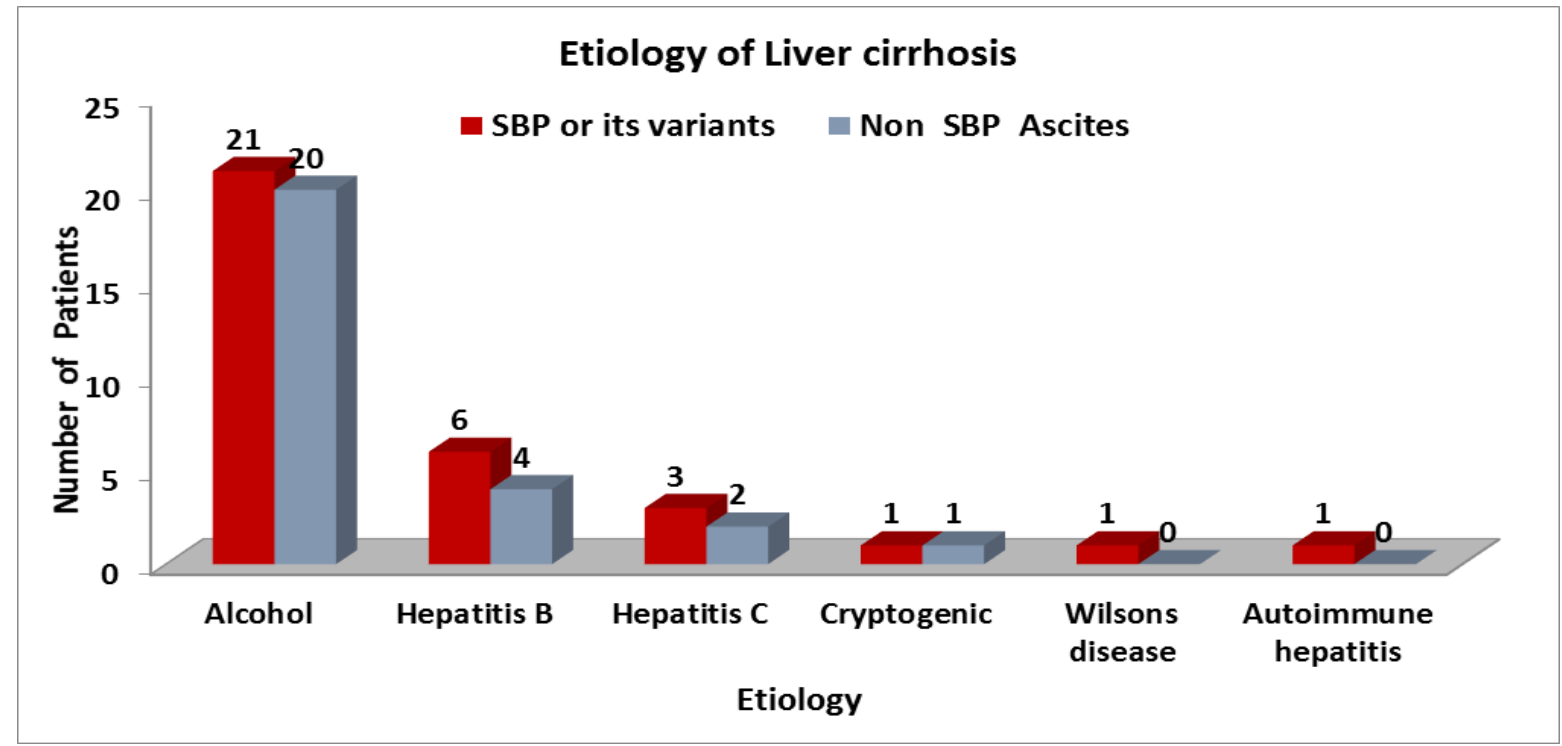

\section{Graph 2}

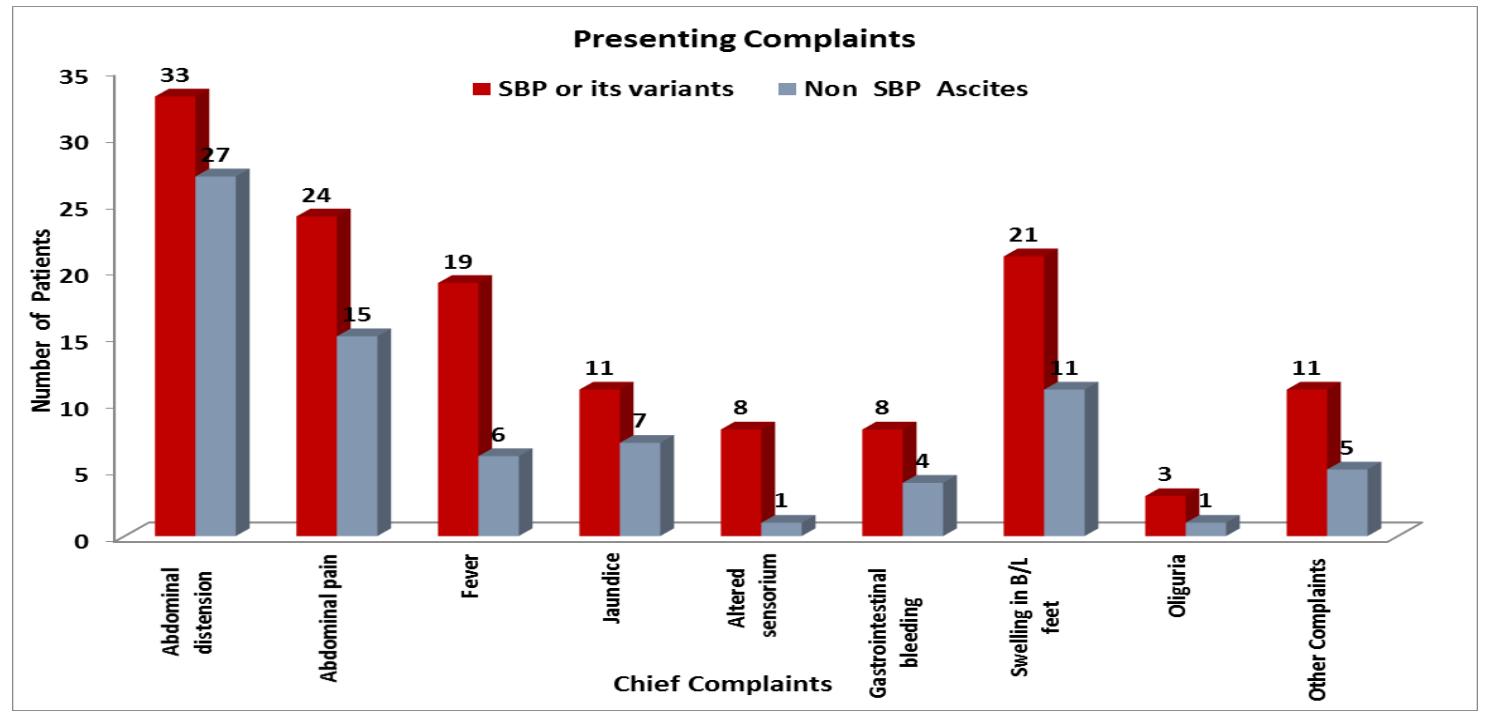

\section{Graph 3}

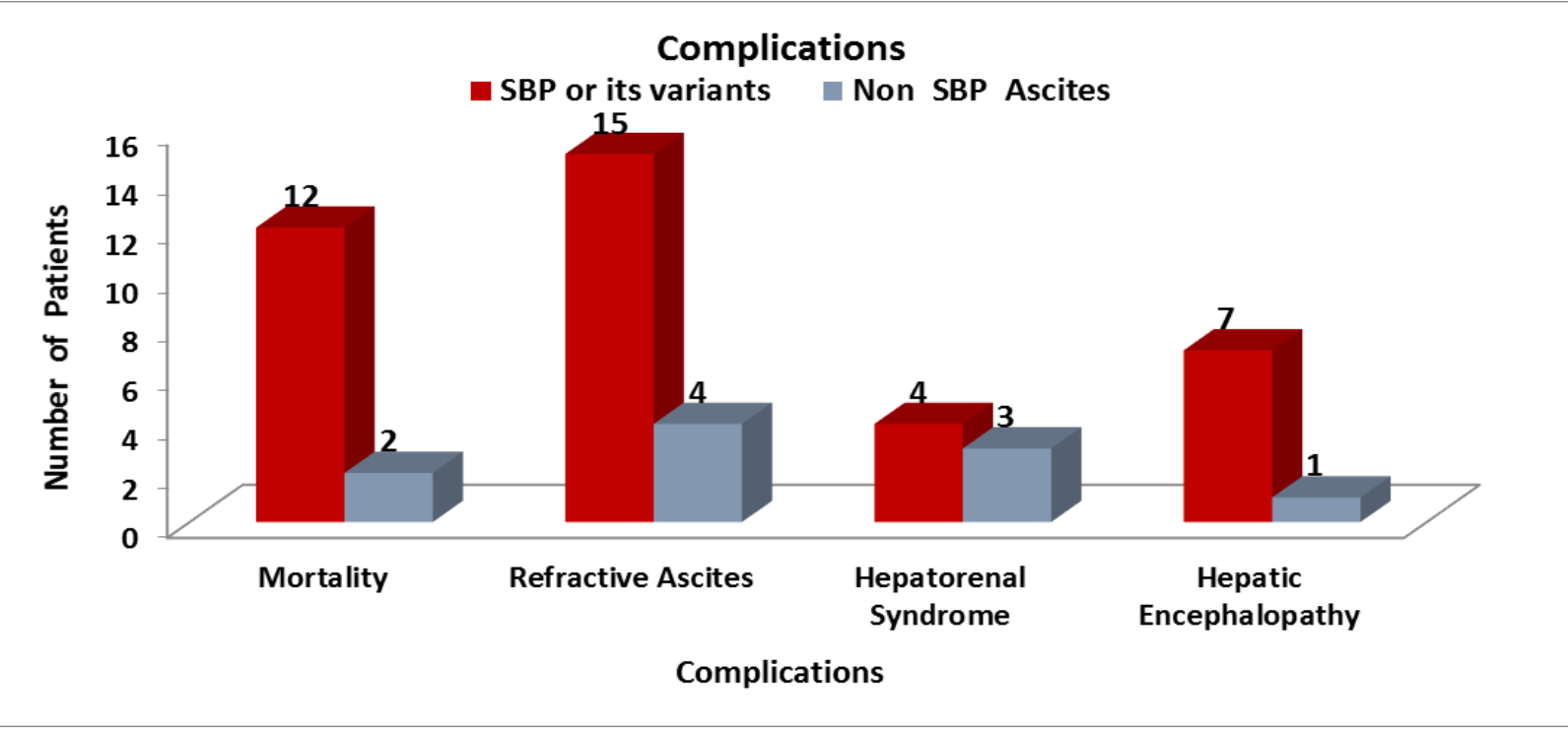


Graph 4:

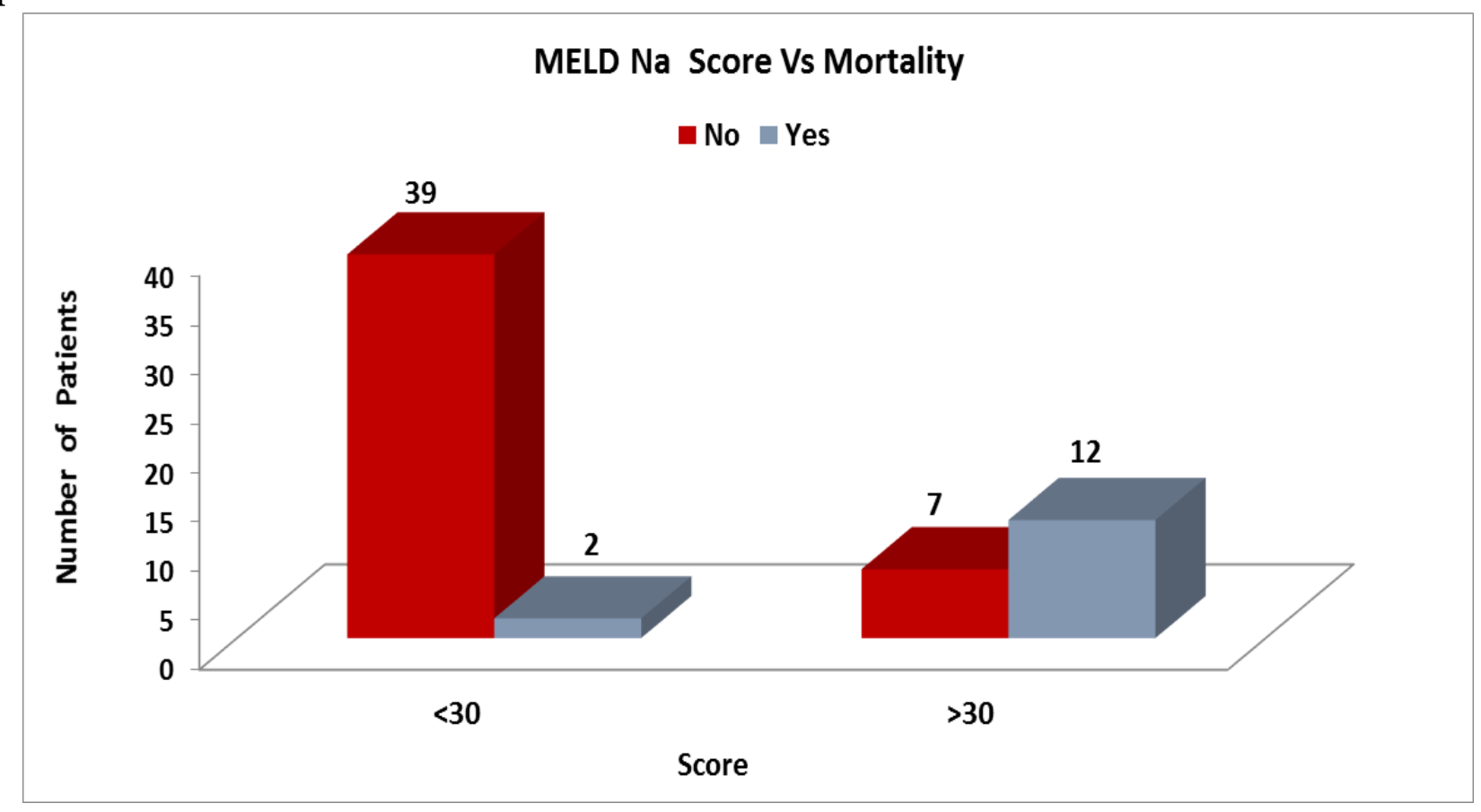

\section{Graph 5}

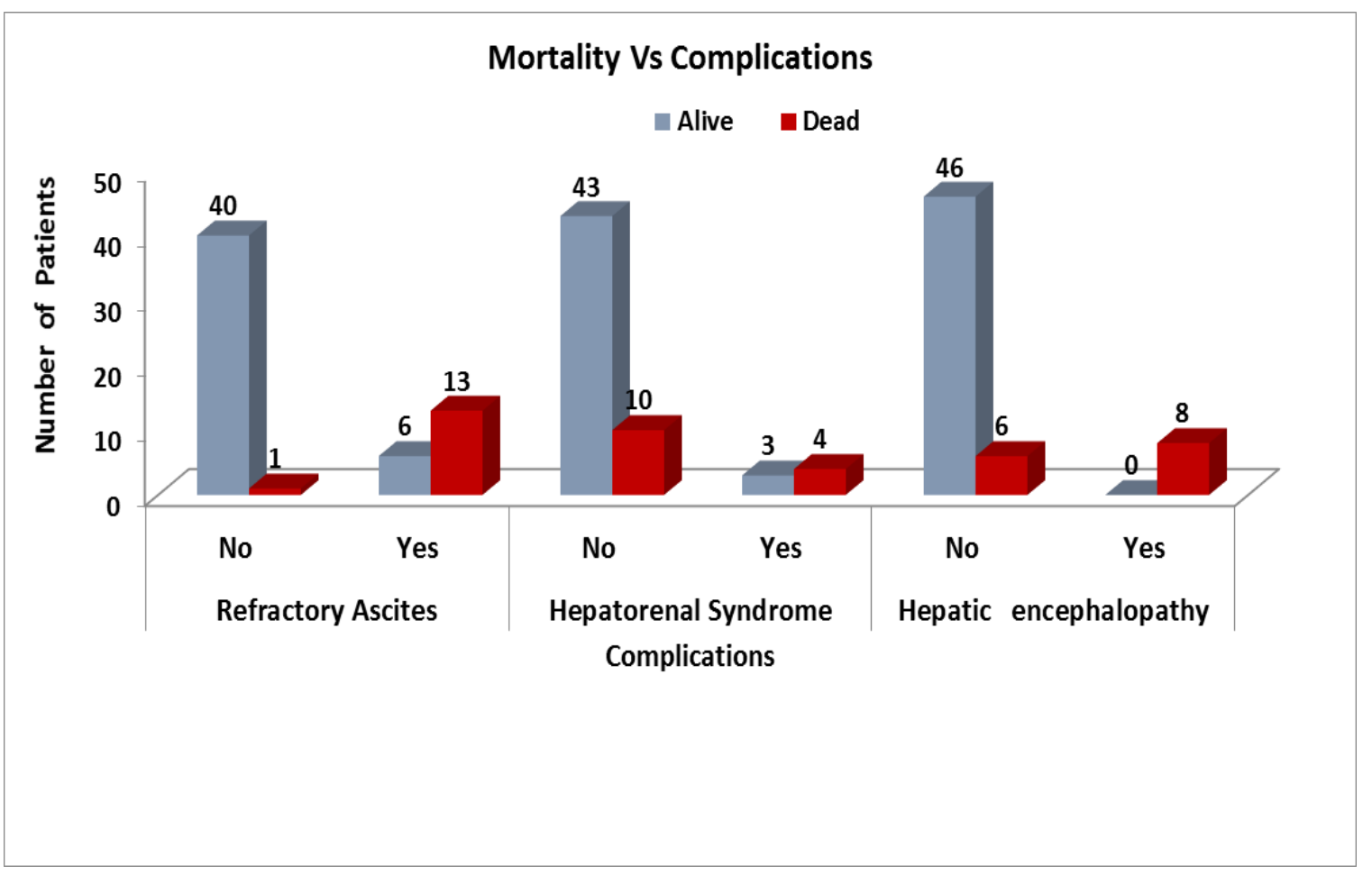

\section{Discussion}

Cirrhosis is a chronic from of liver disease which is usually defined histologically by development of regenerative nodules surrounded by fibrous bands. WHO has estimated that cirrhosis is responsible for $1.1 \%$ of all deaths. Spontaneous Bacterial Peritonitis (SBP) is one of the most frequent, ominous manifestations of decompen- sated liver cirrhosis. Since its initial description in 1964, research has transformed spontaneous bacterial peritonitis (SBP) from a feared disease (with reported mortality of $90 \%$ ) to a treatable complication of decompensated cirrhosis, albeit with steady prevalence and a high recurrence rate. Spontaneous Bacterial Peritonitis is a blood borne infection. Bacteria of gut origin are most 
commonly involved. Extra-intestinal bacteria such as those from the respiratory apparatus, urogenital tract or skin are much less frequently involved. Catheters and other equipment used during invasive procedures represent another possible source of infection ${ }^{1}$. There are four key elements of Spontaneous Bacterial Peritonitis pathogenesis: (1) small intestinal bacterial overgrowth, (2) increased intestinal permeability, (3) bacterial translocation, and (4) immunosuppression. These key elements are not separate, but interlinked. The most common symptoms and signs in patients with spontaneous bacterial peritonitis are pyrexia, increased confusion, diffuse abdominal pain, increasing abdominal girth, vomiting and reduced urine output or ileus. The clinical picture however, is non-specific. In a large number of cases the infection is quite asymptomatic ${ }^{12}$. It is now accepted that the only way to diagnose an episode of spontaneous bacterial peritonitis is by examining a sample of ascitic fluid. It is now recommended that diagnostic paracentesis performed within 12 hours of hospitalization in patients with liver cirrhosis with ascites is associated with an earlier diagnosis of spontaneous bacterial peritonitis and an improved outcome or short term survival since treatment can then be initiated immediately without any delay ${ }^{13}$

Interpretation of Ascitic Fluid: Depending on the ascitic fluid report, diagnosis can be made as spontaneous bacterial peritonitis or its variants i.ebacterascites or culture negative neutrocytic ascites. Spontaneous Bacterial peritonitis is the term used to describe the colonization of ascitic fluid by bacteria isolated on culture together with an increase in ascitic fluid polymorphonuclearcount $(\mathrm{PMNL}) \geq 250 / \mathrm{mm}^{3}$. Bacterascites (monomicrobial non-neutrocyticbacterascites) ${ }^{14}$ is the term used to describe the colonisation of ascitic fluid by bacteria detected on culture, in the absence of inflammatory reaction in the bacterial fluid i.e PMNL count is $<250 / \mathrm{mm}^{3}$. Culture negative neutrocytic ascites (CNNA) ${ }^{15}$ is the term used to describe the clinical situation when the ascitic PMNL count is $\geq 250 / \mathrm{mm}^{3}$ but cultures fail to grow any bacteria. Patients with ascitic fluid PMN counts greater than or equal to 250 cells $/ \mathrm{mm}^{3}$ in a clinical setting compatible with ascitic fluid infection should receive empiric antibiotic therapy. Relatively broad-spectrum therapy is warranted in patients with suspected ascitic fluid infection until the results of susceptibility testing are available. Cefotaxime or a similar third-generation cephalosporin appears to be the treatment of choice for suspected SBP; it covers $95 \%$ of the flora including the 3 most common isolates: Escherichia coli, Klebsiella pneumoniae, and Streptococcal pneumonia. After sensitivities are known, the spectrum of coverage can usually be narrowed. Our study was comparable in parts with the studies conducted by Kavita Paul et al., Nakul Kadam et al. and Chinmaya Kumar Bal Et Al. Our study demonstrated an in hospital mortality of $21.67 \%$ amongst all patients and $20 \%$ amongst patients with SBP or its variants similar to the study by Nakul Kadam et al (17\%). Study by Chinmaya Kumar et al showed a higher mortality rate $(43 \%)$. Our study revealed that variables such as Child Pugh Grade C, MELD Na score > 30, anemia, leukocytosis, coagulopathy or deranged PT INR, hyperbilirubinemia, hyponatremia, AKI, hepatic encephalopathy, refractory Ascites, hepatorenal Syndrome and absence of albumin transfusion during hospitalization were significantly associated with mortality.

\section{Conclusion}

Our study tries to analyse the incidence and the varied clinical and bacteriological profile of patients with SBP or its variants. Although there have been multiple studies on this topic in the past, our study is one of the rare ones which individually correlates various variables as predictors for the development of SBP or its variants and factors that are associated with in hospital mortality in such patients. However, being a small sample study, the results cannot be implemented on a large scale population. 


\section{References}

1. Jan Lata, Oldřich Stiburek, and Marcela Kopacova. Spontaneous bacterial peritonitis: A Severe complication of liver cirrhosis. World J Gastroenterol. 2009 Nov 28; 5(44) 5505-5510.

2. Sherlock S, Dooley J. Spontaneous bacterial peritonitis. In: Sherlock and Dooley's Diseases of the liver and biliary system. 11th ed. Oxford: Blackwell, 2002132-134.134

3. Bacon Bruce R. 17th Edition. 2008. Cirrhosis and Its complications. Harrison's Principles of Internal Medicine; p. 1979.

4. Conn HO. In: Diseases of the liver. 4th edition. Vol. 833. Philadelphia: J.B. Lippincott Company; 1975. Cirrhosis. Edited by Schiff L.

5. MNavasa, J Rodes. Bacterial infections in cirrhosis. Liver Int. 2004; 24:277-80.

6. J Llach, A Rimola, M Navasa, P Ginès, JM Salmerón, A Ginès, et al. Incidence and Predictive factors of first episode of spontaneous bacterial peritonitis in cirrhosis with Ascites: relevance of ascitic fluid protein concentration. Hepatology. 1992; 16:724-27.

7. R Wiest, A Krag, A Gerbes Spontaneous bacterial peritonitis: recent guidelines and Beyond; Gut 2012; 61:297-310 Published Online First: 6 December 2011.

8. Koulaouzidis A, Bhat S, Karagiannidis A, Tan WC, Linaker BD. Spontaneous bacterial Peritonitis; Postgrad Med J. 2007 Jun; 83(980):379-83. 3

9. G Pinzallo, RG Simonetti, ACraxi, SO Piazza, C Spano, 1 Pagliaro. Spontaneous Bacterial peritonitis: A prospective investigation in predominantly nonalcoholic cirrhotic Patients. Hepatology. 1983; 3:545-49.

10. AP Jain, LS Chandra, S Gupta, et al. Spontaneous Bacterial Peritonitis in liver cirrhosis with ascites. J Assoc physicians India. 1999;47(6):619-21.
11. Runyon BA. Ascites and spontaneous bacterial peritonitis. In: Feldman M, Friedman LS,Sleisenger MH, editors. Sleisenger and Fordran's Gastrointestinal and Liver Disease, $8^{\text {th }}$ ed. Philadelphia, PA: Saunders; 2006. pp. 1935-1964.

12. Runyon B A. Paracentesis of ascitic fluid: a safe procedure. Arch Intern Med 19861462259-2261.2261.

13. Song J Y, Jung S J, Park C W. et al Prognostic significance of infection acquisition sitesin spontaneous bacterial peritonitis: nosocomial vs. community acquired. J Korean Med Sci 200621666 671.67.

14. Grabau C M, Crago S F, Hoff LK et al. Performance standards for therapeutic abdominal paracentesis. Hepatology 200440484-488.

15. Lin C H, Chen S C, Ko P C. Pre-procedure coagulation tests are un-necessary before abdominal paracentesis in emergency departments. Hepatology $200541402-$ 403.403. 